\title{
Identifikationsverfahren zur Analyse von EEG-Signalen bei Epilepsie mit Reaktions-Diffusions Netzwerken
}

\author{
F. Gollas ${ }^{1}$ and R. Tetzlaff ${ }^{1}$ \\ ${ }^{1}$ Institut für Angewandte Physik, Johann Wolfgang Goethe-Universität, Frankfurt, Deutschland
}

Zusammenfassung. Partielle Differentialgleichungen des Reaktions-Diffusions-Typs beschreiben Phänomene wie Musterbildung, nichtlineare Wellenausbreitung und deterministisches Chaos und werden oft zur Untersuchung komplexer Vorgänge auf den Gebieten der Biologie, Chemie und Physik herangezogen. Zellulare Nichtlineare Netzwerke (CNN) sind eine räumliche Anordnung vergleichsweise einfacher dynamischer Systeme, die eine lokale Kopplung untereinander aufweisen. Durch eine Diskretisierung der Ortsvariablen können Reaktions-Diffusions-Gleichungen häufig auf CNN mit nichtlinearen Gewichtsfunktionen abgebildet werden. Die resultierenden Reaktions-Diffusions-CNN (RD-CNN) weisen dann in ihrer Dynamik näherungsweise gleiches Verhalten wie die zugrunde gelegten Reaktions-DiffusionsSysteme auf. Werden RD-CNN zur Identifikation neuronaler Strukturen anhand von EEG-Signalen herangezogen, so besteht die Möglichkeit festzustellen, ob das gefundene Netzwerk lokale Aktivität aufweist. Die von Chua eingeführte Theorie der lokalen Aktivität Chua (1998); Dogaru und Chua (1998) liefert eine notwendige Bedingung für das Auftreten von emergentem Verhalten in zellularen Netzwerken. Änderungen in den Parametern bestimmter RD-CNN könnten auf bevorstehende epileptische Anfälle hinweisen. In diesem Beitrag steht die Identifikation neuronaler Strukturen anhand von EEG-Signalen durch Reaktions-DiffusionsNetzwerke im Vordergrund der dargestellten Untersuchungen. In der Ergebnisdiskussion wird insbesondere auch die Frage nach einer geeigneten Netzwerkstruktur mit minimaler Komplexität behandelt.

Correspondence to: $\mathrm{F}$. Gollas

(f.gollas@iap.uni-frankfurt.de)

\section{Einleitung}

\subsection{Reaktions-Diffusions-Systeme}

Vielfältige komplexe Phänomene wie speziell die Ausbreitung elektrischer Nervenimpulse in der Biologie (FitzHugh, 1969) oder oszillierende Reaktionen in der Chemie (Tyson, 1976) sowie generelle Phänomene wie die Bildung von Turing Mustern (Turing, 19952; Murray, 1989) werden durch partielle Differentialgleichungen des Reaktions-Diffusions Typs beschrieben. Die allgemeine Form eines ReaktionDiffusions Systems (Chua, 1998) wird durch

$$
\frac{\partial}{\partial t} \mathbf{x}=\mathbf{f}(\mathbf{x})+\mathbf{D} \nabla^{2} \mathbf{x}
$$

gegeben. Dabei ist die Zustandsvariable $\mathbf{x}(r, t)$ eine $m$ dimensionale Funktion der Raumkoordinaten $\mathbf{r}=r_{1}, \ldots, r_{n}$ und der Zeit $t$. Der Reaktionsteil wird allgemein durch eine nichtlineare Funktion $\mathbf{f}(\cdot)$ mit $\mathbf{f}: \mathbb{R}^{m} \rightarrow \mathbb{R}^{m}$ repräsentiert. Der Diffusionsteil wird durch die zweite räumliche Ableitung, gegeben durch den Laplaceoperator

$\nabla^{2} \mathbf{x}(\mathbf{r}, t)=\frac{\partial^{2} \mathbf{x}(\mathbf{r}, t)}{\partial r_{1}^{2}}+\frac{\partial^{2} \mathbf{x}(\mathbf{r}, t)}{\partial r_{2}^{2}}+\ldots+\frac{\partial^{2} \mathbf{x}(\mathbf{r}, t)}{\partial r_{n}^{2}}$

und eine lineare Gewichtung mit den Diffusionkoeffizienten als Elementen der $m \times m$ Diagonalmatrix D repräsentiert.

\subsection{Reaktions-Diffusions Zellulare Nichtlineare Netzwerke}

Zellulare Nichtlineare Netzwerke (CNN), wie sie in Chua und Yang (1988) vorgeschlagen wurden, sind eine räumliche Anordnung lokal verknüpfter dynamischer Systeme, die als

Published by Copernicus Publications on behalf of the URSI Landesausschuss in der Bundesrepublik Deutschland e.V. 
Zellen bezeichnet werden. Die zeitliche Entwicklung des Zustands einer Zelle kann durch eine gewöhnliche Differentialgleichung - der Zellzustandsgleichung - beschrieben werden (Chua, 1998; Tetzlaff, 2006). Damit ist das Netzwerk als Ganzes durch ein System gekoppelter gewöhnlicher Differentialgleichungen zusammen mit Anfangswerten und Randbedingungen definiert.

Um ein System partieller Differentialgleichungen (1) auf ein $\mathrm{CNN}$ abzubilden, müssen die räumlichen $\mathrm{Ab}$ leitungen diskretisiert werden, was zu einer DiffusionsZellkopplung in der Zustandsgleichung führt. Durch eine finite Differenzen-Approximation des Laplaceoperators $\nabla^{2}$ wird dieser auf eine diskretisierte Version $\tilde{\nabla}^{2}$ abgebildet:

$\nabla^{2} \mathbf{x}(\mathbf{r}) \rightarrow \tilde{\nabla}^{2} x_{\mathbf{r}} \equiv \sum_{\mathbf{k}=\in \mathcal{N}(\mathbf{r})}^{1} A_{\mathbf{k}-\mathbf{r}} x_{\mathbf{k}}$

wobei $\mathcal{N}(\mathbf{r})$ die Nachbarschaft der Zelle an Position $\mathbf{r}$ mit dem Zellzustand $x_{\mathbf{r}}$ und $A$ das Laplace CNN-Template angibt, das durch

$$
\begin{array}{ll}
n=1: & A=\left[\begin{array}{rrr}
1 & -2 & 1
\end{array}\right] \\
n=2: & A=\left[\begin{array}{rrr}
0 & 1 & 0 \\
1 & -4 & 1 \\
0 & 1 & 0
\end{array}\right]
\end{array}
$$

im ein- bzw. zweidimensionalen Fall gegeben ist. Mit Hilfe von Gl. (3) kann ein partielles Differentialgleichungssystem mit 2 Differentialgleichungen (1) durch ein 2-schichtiges $\mathrm{RD}-\mathrm{CNN}$ mit den Zustandsgleichungen

$$
\begin{aligned}
& \dot{x}_{\mathbf{r}}^{1}=f^{1}\left(x_{\mathbf{r}}^{1}, x_{\mathbf{r}}^{2}\right)+D^{1} \tilde{\nabla}^{2} x_{\mathbf{r}}^{1} \\
& \dot{x}_{\mathbf{r}}^{2}=f^{2}\left(x_{\mathbf{r}}^{1}, x_{\mathbf{r}}^{2}\right)+D^{2} \tilde{\nabla}^{2} x_{\mathbf{r}}^{2}
\end{aligned}
$$

angenähert werden, wobei $\dot{x}_{\mathbf{r}}^{\ell}$ den Zellzustand einer Zelle an Position $\mathbf{r}$ in Schicht $\ell$ bezeichnet. Die Diffusionskoeffizienten $D^{\ell}$ der Schicht $\ell$ können mit den Diagonalelementen der Matrix $D$ in Gl. (1) identifiziert werden und die Komponenten des Reaktionsteils in Gl. (1) finden als nichtlineare Kopplungsfunktionen $f^{\ell}$ Eingang in die Zustandsgleichungen des RD-CNN (Gl. 5).

\subsection{RD-CNN mit polynomialen Gewichtsfunktionen}

Um ein RD-CNN gemäß Gl. (5) in numerischen Simulationen zu untersuchen oder um eine schaltungstechnische Realisierung für RD-CNN zu entwerfen ist eine - möglichst allgemeine - Repräsentation der nichtlinearen Gewichtsfunktionen $f^{\ell}$ zu finden. Nimmt man bei einem 2-schichtigen Netzwerk

$f^{\ell}\left(x_{\mathbf{r}}^{1}, x_{\mathbf{r}}^{2}\right)=f_{\ell^{\prime}-\ell=0}^{\ell}\left(x_{\mathbf{r}}^{\ell^{\prime}=\ell}\right)+f_{\ell^{\prime}-\ell}^{\ell}\left(x_{\mathbf{r}}^{\ell^{\prime} \neq \ell}\right)$ an so kann $f_{\ell^{\prime}-\ell}^{\ell}$ durch eine eindimensionale Reihenentwicklung approximiert werden. In diesem Beitrag soll eine Taylor Entwicklung der Ordnung $P$ um Null

$f_{\ell^{\prime}-\ell}^{\ell}\left(x_{\mathbf{r}}^{\ell}\right)=\mathcal{P}_{\ell, \ell^{\prime}}\left(x_{\mathbf{r}}^{\ell^{\prime}}\right) \equiv \sum_{p=0}^{P} \hat{b}_{\ell \ell^{\prime}}^{(p)}\left(x_{\mathbf{r}}^{\ell^{\prime}}\right)^{p}$

mit den Koeffizienten

$\left.\hat{b}_{\ell \ell^{\prime}}^{(p)} \equiv \frac{1}{p !} \frac{d^{p} f_{\ell^{\prime}-\ell}^{\ell}\left(x_{\mathbf{r}}^{\ell}\right)}{d x_{\mathbf{r}}^{\ell p}}\right|_{0}$.

eingeführt werden. CNN mit polynomialen Gewichtsfunktionen $\mathcal{P}_{\ell, \ell^{\prime}}\left(x_{\mathbf{r}}^{\ell^{\prime}}\right)$ können damit eine große Klasse von Reaktions-Diffusions-Systemen beschreiben und sind bereits erfolgreich bei der Modellierung nichtlinearer Systeme betrachtet worden (Puffer et al., 1996). Ein CNN Cellular Wave Computer mit nichtlinearen, polynomialen Gewichtsfunktionen wurde kürzlich in Leiho (2003) vorgestellt.

Mit Hilfe der polynomialen Repräsentation nichtlinearer Gewichtsfunktionen kann die Definition von RD-CNN gemäß Gl. (5) im Blick auf nichtlinearer Nachbarschaftskopplung erweitet werden. So ist etwa eine nichtlineare Diffusion wie im Perona Malik Modell ein erfolgreich verwendeter Ansatz im Bereich der Bildverarbeitung (Gilli et al., 2006). Mit Gl. (7) und Gl. (3) läßt sich ein nichtlineares Nachbarschafts-Kopplungs-Gesetz für den eindimensionalen Fall als

$\mathcal{P} \rightleftharpoons\left(x_{r}\right) \equiv \sum_{p=0}^{P_{D}} D^{(p)}\left(x_{r+1}\right)^{p}+\sum_{p=0}^{P_{D}} D^{(p)}\left(x_{r-1}\right)^{p}$

definieren, wobei $D^{(p)}$ einen Koeffizienten für den Polynom$\operatorname{grad} p$ angibt. In Fällen, in denen keine detaillierten Informationen über die nichtlinearen Kopplungs- und Gewichtsfunktionen vorhanden sind oder aus dem zugrunde liegenden Modell abgeleitet werden könnten, können die Parameter $\hat{b}_{\ell \ell^{\prime}}^{(p)}$ und $D^{(p)}$ in einem überwachten Lernverfahren bestimmt werden.

\section{Identifizierung von EEG-Signalen durch Zell- ausgänge von RD-CNN}

Die Analyse von Elektroencephalogramm - (EEG) - Signalen von Patienten mit Epilepsie steht im Vordergrund zahlreicher Veröffentlichungen (Tetzlaff et al., 2005; Lehnertz und Elger, 1998; Gollas et al., 2004; Tetzlaff und Hein, 2005; Gollas und Tetzlaff, 2005a,b). Mit Methoden der Signalanalyse werden die EEG-Signale des Gehirns untersucht, um bestimmte neurophysiologische Zustände und Störungen zu diagnostizieren. Das so genannte Epilepsie-Problem, d.h. die Erkennung von Anzeichen, die einem epileptischen Anfall vorausgehen, und inhärenter Veränderungen in der hirnelektrischen Aktivität, ist noch nicht gelöst, und Anfälle können 


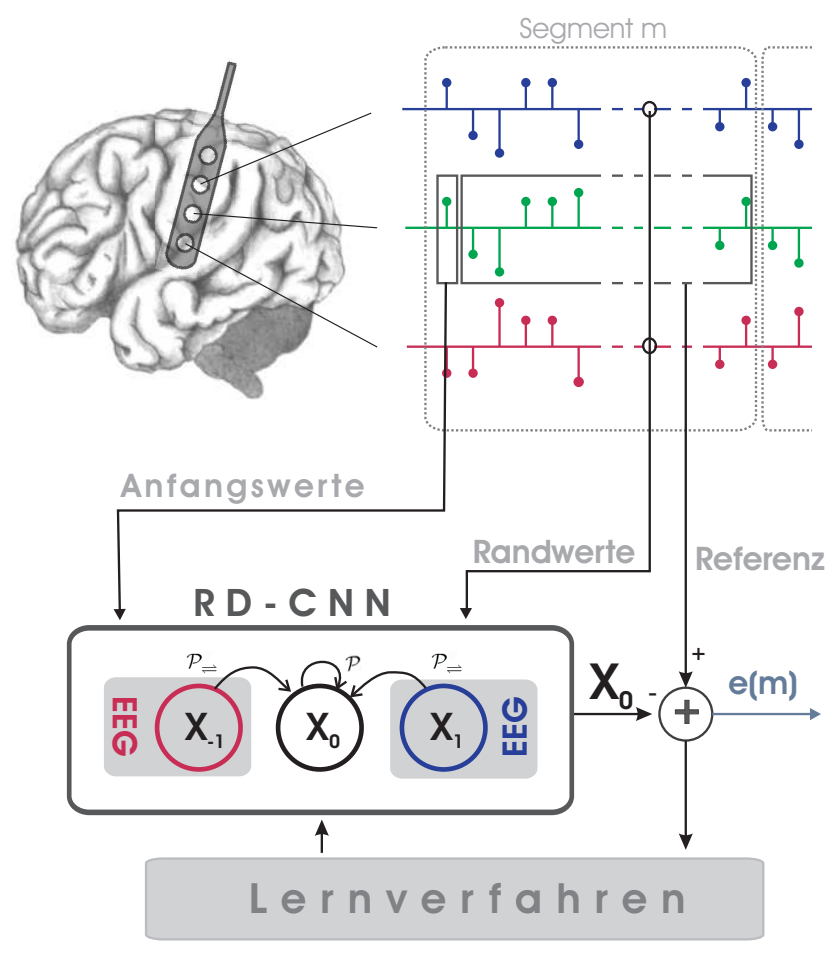

Abb. 1. Approximierung von EEG-Signalwerten durch Zellausgänge eines RD-CNN: In jedem Schritt des Lernverfahrens wird der Fehler (Gl. 11) für ein EEG-Segment berechnet, dabei wird das $\mathrm{zu}$ approximierende Elektroden-Signal (grün dargestellt) und der Zellausgang der Zelle $x_{0}$ herangezogen. Die Netzwerksparameter werden durch das verwendete Trainingsverfahren angepasst bis der Fehler minimal wird. In dem betrachteten RD-CNN koppelt die Zelle mit dem Zustand $x_{0}$ über polynomiale Gewichtsfunktionen an ihre Nachbarzellen und an sich selbst. Die Nachbarzellen werden als Randzellen einbezogen und repräsentieren die EEG-Signal-Werte benachtbarter Elektroden (blau, rot).

noch nicht mit ausreichender Sensitivitvät und Spezifität vorhergesagt werden. Beobachtungen in mehreren neueren Untersuchungen (Lehnertz und Elger, 1998; Gollas et al., 2004) unterstreichen die Bedeutung nichtlinearer Algorithmen in der Analyse von EEGs epileptischer Anfälle. Der Einsatz CNN-basierter Verfahren eröffnet hierbei neue Möglichkeiten. Zum einen können, indem die Dynamik des komplexen, nichtlinearen Systems zur Berechnung herangezogen wird, neue algorithmische Wege beschritten werden. Zum anderen bieten analoge schaltungstechnische Realisierungen den Vorteil hoher Rechenleistung unter Echtzeitbedingungen in Verbindung mit dem Vorteil geringer Leistungsaufnahme und kleiner Größe. Dies läßt CNN-basierte Systeme als mögliche Implantate für den Menschen geeignet erscheinen. Solche mit einer Schnittstelle ausgestatteten Systeme, würden die Möglichkeit einer zeitlich- und örtlich spezifischen medizinischen Behandlung, wie z.B. einer lokalen Injektion eines exakt dosierten Medikaments, oder einer geeigneten elektrischen Stimulation vor Anfallsbeginn, eröffnen.
Mit der Annahme, dass nichtstationäre EEG-Signale durch Ausgangssignale von zeitvarianten komplexen Systemen dargestellt werden können, besteht die Möglichkeit, die Modellparameter im Hinblick auf mögliche Änderungen vor einem epileptischen Anfall zu untersuchen. In diesen Untersuchungen soll festgestellt werden, inwieweit Segmente von EEG-Signalen durch Ausgangswerte von RD-CNN darstellbar sind. Dabei wird das den EEG-Signalen zugrundeliegende neuronale Netzwerk für aufeinanderfolgende Segmente durch eine Folge von RD-CNN identifiziert. Durch die Wahl einer geeigneten Segmentlänge, soll gewährleistet werden, dass für jedes Segment ein zeitinvariantes RD-CNN herangezogen werden kann. Hierbei soll ein einschichtiges RD-CNN

$$
\begin{aligned}
\dot{x}_{r}= & \mathcal{P}_{\ell, \ell}\left(x_{r}\right)+\mathcal{P}_{\rightleftharpoons}\left(x_{r}\right) \\
= & \sum_{p=0}^{P} \check{b}^{(p)} x_{r}^{p}+ \\
& \sum_{p=0}^{P_{D}} D^{(p)}\left(x_{r-1}\right)^{p}+\sum_{p=0}^{P_{D}} D^{(p)}\left(x_{r+1}\right)^{p}
\end{aligned}
$$

mit Gl. (5), (9), und (7) betrachtet werden. In dieser ersten Untersuchung sollen möglichst einfache Netzwerkstrukturen zugrunde gelegt werden. Während der Zustand der Zelle $x_{r=0}$ das berücksichtigte EEG-Elektroden-Signal repräsentiert, liefern die Nachbarelektroden die Signalwerte der Randzellen $x_{+1}, x_{-1}$. Im Rahmen der Parameterbestimmung müssen daher Netzwerke ermittelt werden, deren Ausgangswerte eine minimale Abweichung zu den EEGSignalen aufweisen. Dazu wurde jeweils in einem überwachten Lernverfahren der Wert des relativen, mittleren quadratischen Fehlers

$e(m)=\frac{1}{N} \sum_{t} \frac{(\chi-x)^{2}}{\bar{\chi}^{2}}$

für ein Segment $m$ minimiert (siehe Abb. 1). Dabei bezeichnet $x$ den Zellzustand der Zelle des RD-CNN, das EEGSignalwerte $\chi$ approximiert.

\section{Ergebnisse}

Die Simulation des Netzwerks wurde mit der CNNSimulationssoftware "SCNN" durchgeführt (Ames et al., 2000; Loncar et al., 2000), und dabei eine Runge-Kutta Integration 4. Ordnung verwendet. Das Parametertraining wurde jeweils mit normal verteilten Startwerten begonnen.

Die ausgewerteten EEG-Signale stammen aus einer invasiven Langzeit-EEG-Ableitung, welche im Rahmen prächirurgischer Diagnostik eines männlichen Patienten mit fokaler Epilepsie in der Klinik für Epileptologie der Universität Bonn gewonnen wurde. Die Aufzeichnung umfasst einen zusammenhängenden Bereich von 6 Tagen. Die Werte stammen von 48 Elektroden, die mit $200 \mathrm{~Hz}$ abgetastetet wurden. Im Zeitraum der Aufzeichnung wurden 10 klinische Anfälle verzeichnet. 


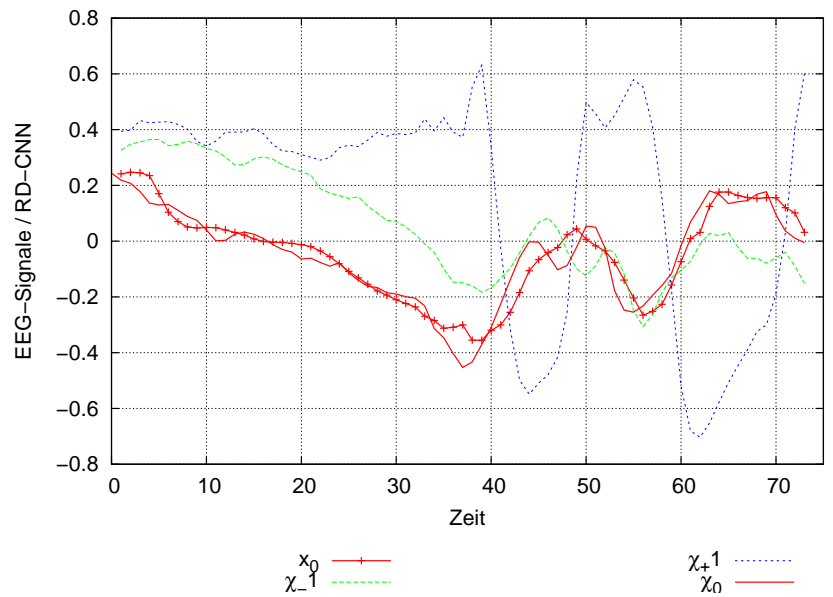

Abb. 2. Zellausgänge $x_{0}$ des RD-CNN gemäß (10) mit $P=8$ und $P_{D}=8$, EEG-Elektroden-Signal $\chi_{0}$, das als Referenz verwendet wurde und EEG-Signale $\chi_{-1}, \chi_{+1}$ benachbarter Elektroden, die als Randwerte dienten. (Segmentlänge $t=75$, minimaler Fehler $e(m)=0.2567)$

Tabelle 1. Fehler $e(m)$ gemittelt über 20 zufällig gewählte Segmente und berechnet mit verschiedenen Lernverfahren nach Ames et al. (2000); Teukolsky et al. (1992).

\begin{tabular}{cc}
\hline Lernverfahren & $\overline{\mathbf{e}(\mathbf{m})}$ \\
\hline Simulated Annealing & 0.2253 \\
Simplex & 0.3096 \\
Iterative Annealing & 0.3565 \\
Genetic & 0.3680 \\
RPLA & 0.3812 \\
Powell & 1.3067 \\
RBP & $\infty^{1}$ \\
BFGS & $\infty^{1}$ \\
Conjugated Gradient & $\infty^{1}$ \\
\hline
\end{tabular}

Zunächst wurden verschiedene Lernverfahren zur Minimierung des Fehlers im vorgestellten Verfahren betrachtet. Für jedes Lernverfahren wurde der kleinste erreichte Fehler $e(m)$ für 20 zufällig ausgewählte Segmente bestimmt. Der über diese 20 Segmente gemittelte Fehler ist in Tab. 1 verzeichnet. Im Vergleich der untersuchten Optimierungsverfahren zeigt "Simulated Annealing" das beste Resultat, während es bei gradientenbasierten Verfahren in vielen Fällen zu keiner Konvergenz für einen minimalen Fehler kam.

Desweiteren wurde der Effekt der Wahl der Höhe der Polynomgrade $P$ und $P_{D}$ der polynomialen Kopplungs- und Gewichtsfunktionen auf die Genauigkeit der Approximierung untersucht. Das Ergebnis ist in Tab. 2 verzeichnet. Da-

\footnotetext{
${ }^{1}$ Für eine große Zahl von Segmenten konnte durch das Lernverfahren kein minimaler Fehler $e(m)$ bestimmt werden.
}

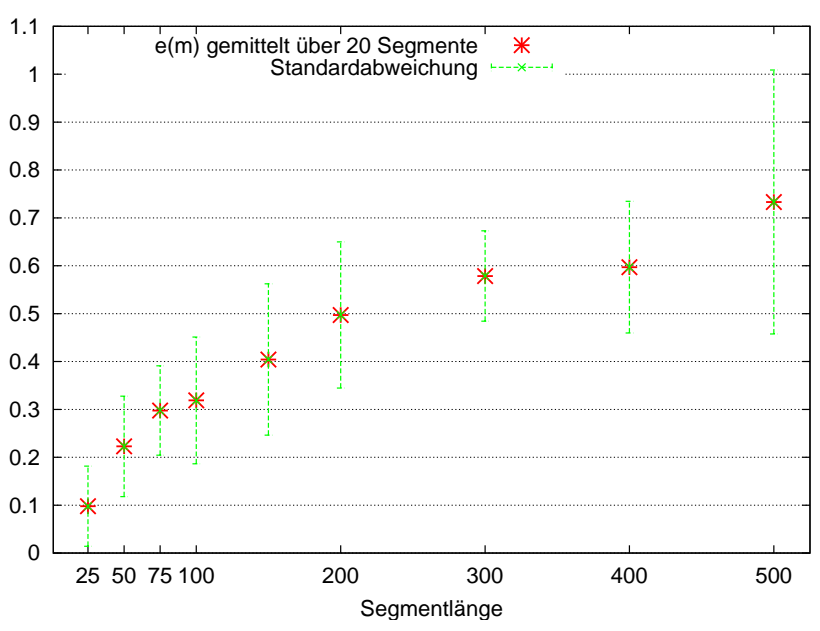

Abb. 3. Über 20 Segmente gemittelter Fehler gemäß (11) aufgetragen gegen die Länge der betrachteten Segmente.

bei wurde wiederum der Fehler $e(m)$ über 20 Segmente, mit der Länge $t=50$, gemittelt und es wurde das "Simulated Annealing" Verfahren (Teukolsky et al., 1992) gewählt. Die Ergebnissen lassen erkennen, dass nicht nur die Verwendung von Gewichtsfunktionen höherer Ordnung im Reaktionsteil des RD-CNN (Eq. 10), sondern auch Nachbarschaftskopplungsfunktionen höherer Ordnung die Genauigkeit des vorgeschlagenen Modellierungsverfahrens verbessern.

Ferner wurde der minimalen Fehler $e(m)$ bei unterschiedlichen Segmentlängen bestimmt. Dabei wurden jeweils 20 Segmente mit einer Länge von $t=50$ bis zur Länge von $t=500$ untersucht. In Abb. 3 sind die gemittelten Fehler entsprechend Gl. (11) gegen die Segmentlänge aufgetragen. Man findet, dass bei größeren Segmentlängen ebenfalls höhere Werte für den Fehler $e(m)$ auftreten.

Abbildungen 2 und 4 zeigen beispielhaft das Ergebnis der Identifikation von EEG-Signalen durch Zellausgänge des RD-CNN. In Abb. 2 sind die Zellausgangswerte der durch das beschriebene Parameteroptimierungsverfahren ermittelten RD-CNNs und das Referenz-EEG-Signal sowie die als Randwerte verwendeten EEG-Signale für ein Segment der Länge $t=75$ aufgetragen. Es ist zu erkennen, dass die EEGSignalform durch das eingeführte, einfache RD-CNN Modell gut approximiert wird.

Abbildung 4 zeigt im Bereich $t=201 \cdots 500$ die Zellausgänge des für das vorausgehende Segment $t=1 \cdots 200$ trainierte RD-CNN gegenüber den nachfolgenden EEGSignalwerten, die während des Optimierungsverfahrens nicht berücksichtigt wurden. Auch hier läßt sich für das betrachtete RD-CNN eine gute Annäherung der Zellausgänge des Modells an die EEG-Signalwerte festellen, die nicht zur Bestimmung des Modells herangezogen wurden. 
Tabelle 2. Fehler $e(m)$ gemittelt über 20 Segmente ermittelt mit verschiedenen polynomialen Ordnungen gemäß Gl. (7) und (9).

\begin{tabular}{ccccccccc}
\hline & $P_{D}=1$ & $P_{D}=2$ & $P_{D}=3$ & $P_{D}=4$ & $P_{D}=5$ & $P_{D}=6$ & $P_{D}=7$ & $P_{D}=8$ \\
\hline$P=1$ & 0.553060 & 0.407094 & 0.371738 & 0.373991 & 0.334633 & 0.308310 & 0.288700 & 0.273802 \\
$P=2$ & 0.427972 & 0.373961 & 0.329795 & 0.334265 & 0.292390 & 0.290451 & 0.281247 & 0.267392 \\
$P=3$ & 0.366183 & 0.323929 & 0.295558 & 0.287398 & 0.276862 & 0.277051 & 0.276716 & 0.243809 \\
$P=4$ & 0.392346 & 0.326408 & 0.288364 & 0.258347 & 0.264322 & 0.275421 & 0.258108 & 0.262506 \\
$P=5$ & 0.356876 & 0.296847 & 0.302006 & 0.278714 & 0.276380 & 0.247402 & 0.231866 & 0.235688 \\
$P=6$ & 0.342840 & 0.281476 & 0.275603 & 0.268526 & 0.256282 & 0.262545 & 0.223685 & 0.236095 \\
$P=7$ & 0.325110 & 0.292070 & 0.273210 & 0.240251 & 0.256868 & 0.224650 & 0.257639 & 0.234809 \\
$P=8$ & 0.333395 & 0.280435 & 0.260762 & 0.238250 & 0.230291 & 0.240105 & 0.229042 & 0.222546 \\
\hline
\end{tabular}

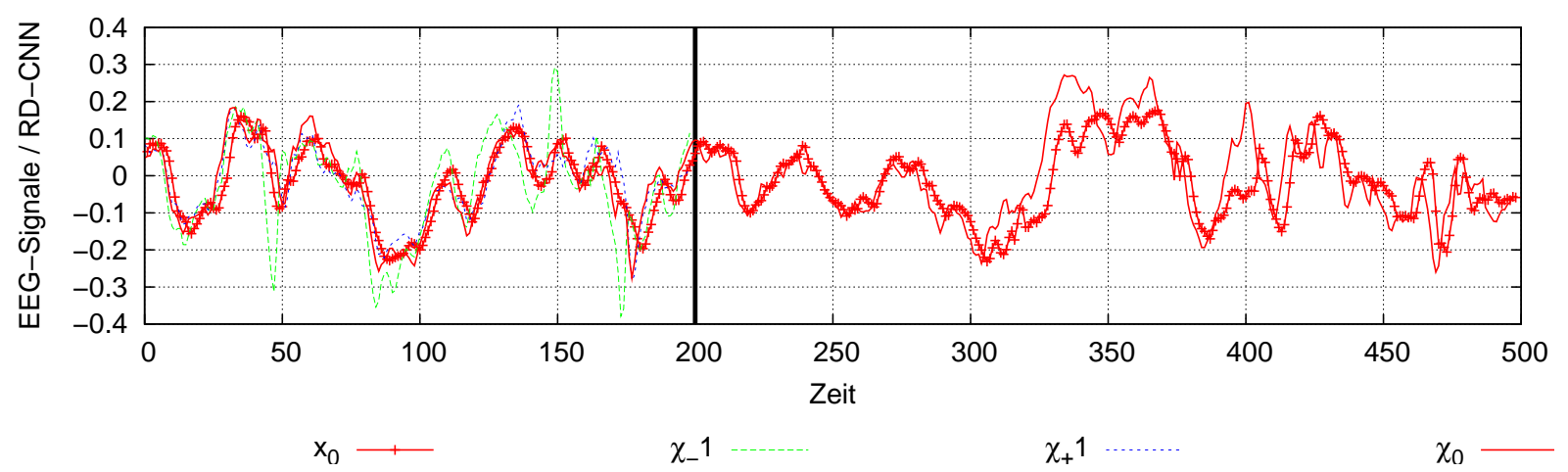

Abb. 4. Zellausgänge $x_{0}$ des RD-CNN gemäß Gl. (10) mit $P=3, P_{D}=1, e(m)=0.395$ EEG-Elektroden-Signal $\chi_{0}$, das als Referenz verwendet wurde und EEG-Signale $\chi_{-1}, \chi_{+1}$ von benachbarten Elektroden. Für das Parametertraining wurde das Segment von $t=1 \cdots 200$ verwendet, um den Bereich $t=201 \cdots 500$ (keine Optimierung) zu identifizieren.

\section{Zusammenfassung}

In diesem Beitrag wurden Reaktions-Diffusions Zellulare Nichtlineare Netzwerke (RD-CNN) mit polynomialen Gewichtsfunktionen und nichtlinearen Nachbarschaftskopplungsfunktionen vorgeschlagen, um kurze Segmente von EEG-Signalen bei Epilepsie zu approximieren. Dabei werden die Parameterwerte in einem überwachten Lernverfahren bestimmt. Die Einflüsse verschiedener Segmentlängen, verschiedener Polynomgrade der Gewichtsfunktionen des Reaktionsteils und der Nachbarschaftskopplungen, sowie verschiedener Lernverfahren auf die Genauigkeit der vorgeschlagenen Modellierungsverfahren wurden untersucht. Gegenstand weiterer Untersuchung soll die Verfeinerung des Modells und die Analyse bezüglich der Veränderung von Modellparametern vor epileptischen Anfällen sein.

Danksagung. Diese Arbeiten wurden durch die Deutschen Forschungsgemeinschaft gefördert (Projekt TE257/6-3,4). Für die zur Verfügung gestellten EEG-Daten bedanken wir uns bei Klaus Lehnertz, Klinik für Epileptologie der Universität Bonn und seiner Gruppe.

\section{Literatur}

Ames, C., Feiden, D., Geis, G., Kunz, R., Loncar, A., Puffer, F., Schoenmeyer, R., Tetzlaff, R., and Weiss, D.: SCNN 2000 Dokumentation, Institut für Angewandte Physik, Johann Wolfgang Goethe Universität, Frankfurt am Main, 2000.

Chua, L. O.: A Paradigm of Complexity, World scientific series on nonlinear science, University of California, Berkeley, V. 31 of A, 1998.

Chua, L. O. and Yang, L.: Cellular neural networks: Theory and applications, IEEE Transactions on Circuits and Systems, 35, 1257-1272, 1988.

Dogaru, R. and Chua, L. O.: Edge of chaos and local activity domain of fitzhugh-nagumo equation, International Journal of Bifurcation and Chaos, 8, 211-257, 1998.

FitzHugh, R.: Mathematical models of excitation and propagation nerve, Biol. Eng. H. Schwan. E d. New York, McGraw-Hill, 1969.

Gilli, M., Corinto, F., and Biey, M.: Non-linear coupled cnn models for multiscale image analysis, International Journal of Circuit Theory and Applications, 34, 77-88, 2006.

Gollas, F., Niederhöfer, C., and Tetzlaff, R.: Prediction of brain electrical activity in epilepsy using a higher dimensional prediction algorithm for discrete time cellular neural networks (dtcnn), Circuits and Systems, 2004, ISCAS '04. Proceedings of the 2004 International Symposium, Vancouver, Canada, 6, V-720-V-723, 2004. 
Gollas, F. and Tetzlaff, R.: Modeling brain electrical activity in epilepsy by reaction-diffusion cellular neural networks, Proc. SPIE's Microtechnologies for a New Millenium, Seville, Spain, 2005a.

Gollas, F. and Tetzlaff, R.: Modeling complex systems by reactiondiffusion cellular nonlinear networks with polynomial weight functions, Cellular Neural Networks and Their Applications CNNA, 2005 9th International Workshop, Hsin-Chu, Taiwan, 2005b.

Laiho, M.: Mixed-Mode Cellular Array Processor, Realization for Analyzing Brain Electrical Activity in Epilepsy, PhD thesis, Helsinki University of Technology, Electronic Circuit Design Laboratory, Report 38, 2003.

Lehnertz, K. and Elger, C. E.: Can epileptic seizures be predicted? Evidence from nonlinear time series analyses of brain electrical activity, Phys. Rev. Lett, 80, 5019-5022, 1998.

Loncar, A., Kunz, R., and Tetzlaff, R.: Scnn 2000 - part i: Basic structure and features of the simulation system for cellular neural networks, Proceedings of the International Workshop on Cellular Neural Networks and their Applications, Catania, 2000.

Murray, J. D.: Mathematical Biology, Springer-Verlag, 1989.
Puffer, F., Tetzlaff, R., and Wolf, D.: Modeling nonlinear systems with cellular neural networks, Proceedings of the IEEE International Conference On Acoustics, Speech And Signal Processing (ICASSP 1996), Atlanta, USA, 3513-3516, 1996.

Tetzlaff, R.: Zellulare nichtlineare netzwerke: Anwendungen in der informationstechnologie, Proceedings of Kleinheubacher Tagung, Miltenberg, this issue, 2006.

Tetzlaff, R. and Hein, D. A.: Wavelet based analysis of multielectrode eeg-signals in epilepsy, Proceedings of the Microtechnologies for the New Millenium SPIE Europe 2005, Seville, Spain, 66-74, May, 2005.

Tetzlaff, R., Niederhöfer, C., and Fischer, P.: Intelligent implantable medical devices: the epilepsy problem, Proceedings of SPIE's Microtechnologies for a New Millenium, Sevilla, Spain, 2005.

Teukolsky, S. A., Press, H. W., Flannery, B. P., and Vetterling, W. T.: Numerical recipes in C: The Art of Scientific Computing, Cambridge University Press, New York, 2nd edition edition, 1992.

Turing, A. M.: The chemical basis of morphogenesis, Trans. Roy. Soc. London, 237, 1952.

Tyson, J. J.: The belousov-zhabotinsky reaction, Springer Lecture Notes in Biomathematics, Berlin, 1976. 